\title{
The significance of teaching human biochemistry to dental students
}

\author{
Haseeb Ahsan
}

Department of Biochemistry, Jamia Millia Islamia, Faculty of Dentistry, New Delhi, India

\begin{abstract}
Objectives: Biochemistry instructions in dental studies should highlight the fact that the basic biochemical processes occurring in the oral cavity are the same as those that take place in other tissues and organs of the body. Saliva is a complex fluid that plays an essential role in the maintenance of oral health and contains a wide range of proteins, enzymes (lysozyme, lactoferrin, peroxidases) and secretory immunoglobulins. Salivary cationic peptides and defense proteins, such as lysozyme, salivary amylase, cystatins, mucins, peroxidases, and statherin, are primarily responsible for innate immunity. The role of collagen, which forms a large part of the organic material of dentin, should be given prominence in the study of proteins. The study of carbohydrates and lipids is also an important topic, since the digestion of starch and lipids begins in the oral cavity due to the presence of various enzymes, e.g., amylase and lipase. The biochemistry syllabus for dental sciences should also include the role of weak acids and bases and buffers to help students understand the buffering capacity of saliva and its implications for oral health. Patients with poor glycemic control are more prone to oral manifestations of diabetes, including periodontal disease, salivary gland dysfunction, halitosis, burning mouth sensation, delayed wound healing, and increased susceptibility to infection. Diabetic patients are also at greater risk of an intraoperative diabetic emergency in the dental clinic. Therefore, dentists must appreciate and implement important dental management considerations when providing care to diabetic patients. Strategies to investigate bleeding disorders can help guide stepwise, rational testing for inherited and acquired causes of bleeding. It is clear that biochemistry is becoming increasingly important in the field of dentistry, and this should be reflected in both teaching and research.

Keywords: Biomarkers, biomolecules, clinical biochemistry, dental caries, medical biochemistry, oral cavity, saliva
\end{abstract}

Untors ndergraduate students of medical sciences are required to apply professional and scientific knowledge in competence-based clinical skills acquired through laboratory training and patient interaction. Scientific and technological developments in medical sciences should be included in academic and clinical programs. Advancements in molecular biology, biochemistry, genomics, proteomics, and tissue engineering provide the basis for the use of new technologies in medicine. Laboratory sciences and research education is an important aspect of a health science curriculum and includes biomedical sciences and clinical biochemistry [1]. Knowledge of advanced medical biochemistry and laboratory clinical biochemistry is now an important aspect of medical and surgical disciplines (Fig. 1). The clinical biochemistry contribution of the analysis of glycated hemoglobin (HbA1c) to the diagnosis and monitoring of diabetes mellitus (DM) is particularly valuable. The importance of a serum lipid profile, including cholesterol and lipoproteins, for public health has increased enormously, as it identifies a risk factor for cardiovascular disease. Point-ofcare testing, i.e., the development of a wide range of portable instruments, analyzers, and test kits, which allows for emergency testing of patients in hospitals or self-testing by individuals, was a substantial methodological development [2].

\section{Biochemistry in dentistry}

Medical sciences and therapeutics have progressed enormously over the last half a century, including the subject

Address for correspondence: Haseeb Ahsan, PhD. Department of Biochemistry, Jamia Millia Islamia, Faculty of Dentistry, Jamia Nagar,

New Delhi, 110025, India

Phone: +91-1126982006 E-mail: drhahsan@gmail.com ORCID: 0000-0002-5313-5959

Submitted Date: February 02, 2021 Accepted Date: March 01, 2021 Available Online Date: March 19, 2021

${ }^{\circ}$ Copyright 2021 by International Journal of Medical Biochemistry - Available online at www.internationalbiochemistry.com

OPEN ACCESS This work is licensed under a Creative Commons Attribution-NonCommercial 4.0 International License. 


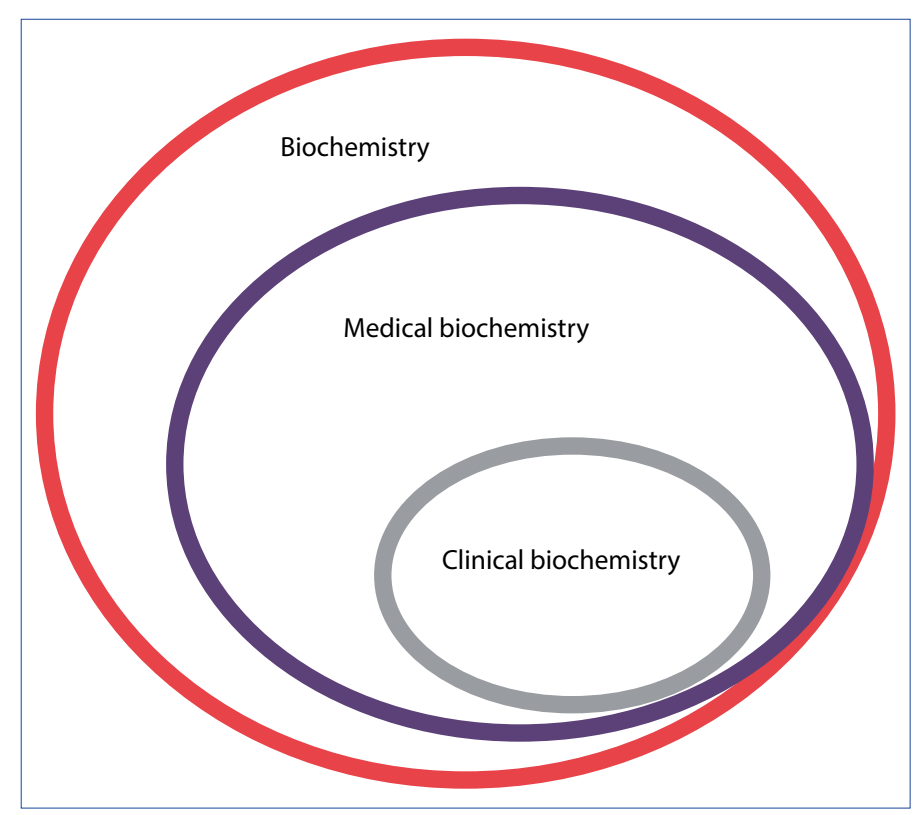

Figure 1. The relationship between general biochemistry, medical biochemistry, and clinical biochemistry (reproduced from Dominiczak, 2011).

area of oral health and dental diseases. The discipline of dentistry or dental hygiene was initially developed in the late $19^{\text {th }}$ century to treat dental caries, but it eventually led to recognition and acceptance for the treatment of diseases of the oral cavity, also called the buccal cavity. It is now an advanced and well-established branch of human health sciences responsible for the treatment and management of diseases of the mouth, teeth, and related tissues. Better oral care and hygiene, advanced dental replacement materials, water fluoridation, and the adoption of drugs used for non-dental diseases have resulted in better dental treatments. The most common dental diseases, i.e., tooth cavities (dental caries) and bleeding gums (periodontal disease), are chronic conditions that are still not fully understood at the molecular and cellular level $[3,4]$. Therefore, molecular biology and biochemistry have become increasing significant in dental sciences in order to understand the cause and pathogenicity of dental diseases [4].

The oral cavity is the uppermost part of the digestive system of human body and is the area of the mouth demarcated by the lips, cheeks, palate, and the floor of the cavity. The main structures of the mouth are the teeth, which tear and grind ingested food into small pieces suitable for digestion; the tongue, which mixes food and has sensory receptors for taste; and the palate, which separates the mouth from the nasal cavity, allowing separate passage of air and food. The favorable environment and the secretions present in the mouth help to soften food, facilitate swallowing, and start the process of digestion [5]. Moreover, oral fluids, such as saliva and oral tissue exudate, known as gingival crevicular fluid (GCF), are important diagnostic tools for the detection of diseases of the oral cavity. GCF is a useful body fluid for the diagnosis of
DM and other inflammatory conditions [1]. Hence, it should be an important aspect of biochemistry to emphasize that the basic biochemical processes occurring in the oral cavity are the same as those that take place in other tissues and organs of the body [4].

\section{Biochemistry of dental caries and plaque}

The study of carbohydrates is an important topic in dental sciences since sugars have an significant role in the health of teeth. In addition, the digestion of carbohydrates (starches) starts in the mouth due to the presence the a-amylase enzyme, which is present in salivary secretions. Carbohydrates, such as polysaccharides, are classified according to their structure and function; storage polysaccharides are grouped as intracellular (e.g., starch and glycogen) or extracellular (e.g., dextran). Dextran is an intracellular polysaccharide found in cariogenic bacteria, while glycogen is present in the liver and skeletal muscles in humans. The study of complex polysaccharides constitutes an important area of interest to dental students, e.g., salivary mucins are glycoproteins, and the connective tissue and the organic matrix of dentine consists of mucopolysaccharides (glycosaminoglycans) [6]. The concentration of dietary sucrose has a significant contribution to dental caries and has the highest cariogenic potential. The Maillard reaction involves the biochemical interactions between sugars and proteins that are responsible for the lesion discoloration in the carious process, known as the browning reaction, categorized as a non-enzymatic glycation process. It involves an irreversible alteration in the amino acid structure leading to a change in protein function. The Maillard reaction leads to the formation of brown polymers called melanoidins, and the severity of disease can be evaluated based on the extent of brown discoloration [7]. Dental caries, or tooth decay, is a dynamic process in which the dental biofilm (microbial deposit) on the tooth surface undergoes metabolic reactions that result in chemical dissolution of the tooth. The frequency of carbohydrate consumption plays a vital role in the carious process. People who consume a large amount of carbohydrates have a lower oral $\mathrm{pH}$ since the buffering capacity of saliva is modified. A pH level of 5.5 is critical for the enamel in the carious process, as a lower $\mathrm{pH}$ indicates that there is net mineral loss of tissue from the tooth. Dentine is composed of more organic material and water, and also degrades in the carious process at a near neutral $\mathrm{pH}(\mathrm{pH}$ 6.2) [7]. A carious tooth is the result of the dissolution of hydroxyapatite $(\mathrm{HA})$ from calcified parts of the tooth accompanied by breakdown of the dentine collagen or enamel amelogenins, since the $\mathrm{pH}$ of the mouth is lowered due to anaerobic breakdown of carbohydrates by oral bacteria [8].

Matrix metalloproteinases (MMPs) are zinc-dependent, host-derived proteolytic enzymes, which are responsible for the degradation of the organic matrix of dentine in the carious process. The $\mathrm{pH}$ change in a carious lesion activates 
MMPs, which function at a neutral $\mathrm{pH}$, since saliva neutralizes an acidic $\mathrm{pH}$, allowing MMPs to become activated and leading to the degradation of the organic matrix of the dentine. The MMPs require zinc, since it influences the signaling pathway of MMPs resulting in dentine remineralization. Therefore, zinc is an important element that can be used as a therapeutic agent for the remineralization of a tooth. Zinc-leaching dental materials, such as amalgams and cements (zinc phosphate, calcium hydroxide, zinc oxide), are thought to inhibit the demineralization of dentine and promote remineralization [7]. The production of organic acids $\left(\mathrm{H}^{+}\right)$as a result of bacterial degradation of sugars causes the $\mathrm{pH}$ of plaque to fall, resulting in the dissolution of $\mathrm{HA}$ into calcium ions, hydrogen phosphate ions and water, thereby leading to demineralization within the enamel. It is well established that fluoride ions can inhibit bacterial growth. It adheres to the enamel, forming fluorohydroxyapatite, which is more resistant to acid attack. The antimicrobial property of fluoride inhibits enzyme enolase in the glycolytic pathway and bacteria are no longer able to thrive and promote tooth demineralization in the carious process [7].

Dental plaque biofilm is composed of diverse microflora found on the surface of teeth. The microorganisms within the biofilm metabolize fermentable carbohydrates in the diet, producing organic acids which dissolve the HA component of teeth. The bacteria are able to withstand and flourish in a low $\mathrm{pH}$ environment, and are subsequently responsible for the development of dental caries [7]. Streptococcus mutans is the principal bacterial species of dental caries and is able to thrive and multiply due to its ability to tolerate an acidic environment. The $S$. mutans glucosyltransferases (GTFs) are important enzymes in the formation and development of dental plaque biofilm. Specific GTFs are upregulated in response to a low $\mathrm{pH}$, aiding in the colonization of other bacteria and the cohesion of plaque, contributing to a caries-favorable environment [7].

\section{Biochemistry of saliva}

Saliva is an important oral fluid that is secreted from the salivary glands and is involved in numerous functions in the mouth, including digestion, swallowing, lubrication, protection, and maintenance of tooth integrity [9-12]. The biochemistry of saliva is an important field of study and the presence of various organic and inorganic components have been identified. Saliva is an exocrine secretion consisting of $95 \%$ to $99 \%$ water and contains electrolytes, proteins, enzymes, immunoglobulins, antimicrobial factors, urea, mucosal glycoproteins, etc. that are important to oral health [13]. Saliva contains a variety of proteins with antibacterial properties and those necessary for inhibiting the spontaneous precipitation of calcium and phosphate ions in the salivary glands and their secretions. The balance of hydrogen-bicarbonate ions in saliva is responsible for the buffering capacity and $\mathrm{pH}$ that protect the enamel from dental caries. The action of buffers such as inorgan- ic phosphate $(\mathrm{Pi})$ and the carbonic acid-bicarbonate $\left(\mathrm{H}_{2} \mathrm{CO}_{3^{\prime}}\right.$ $\mathrm{HCO}_{3}^{-}$) system maintain a neutral $\mathrm{pH}$ in saliva. An increased salivary flow influences the biochemistry of the carious process, as it increases the concentration of bicarbonate, chloride, and sodium ions. The bicarbonate ion concentration neutralizes acids and promotes remineralization of teeth. Therefore, an adequate flow of saliva is necessary for the prevention of dental caries [7].

Sufficient salivary flow and composition are important for the lubrication and protection of oral tissue against desiccation, penetration, ulceration, and potential carcinogens by mucin and anti-proteases [14]. Saliva includes various components, including histatins, anti-bacterial polypeptides, acidic proline-rich proteins (PRPs), mucins, glycoproteins responsible for the viscosity of mucosal secretions, lysozyme, secretory immunoglobulin A (s $\lg \mathrm{A})$, cystatins, a-amylase, kallikreins, etc. [15-17]. The complex polysaccharides unique to the oral cavity include glycoproteins, such as salivary mucins, mucopolysaccharides (glycosaminoglycans) and extracellular polysaccharide (e.g., dextrans) occurring in cariogenic bacteria [8]. The oral cavity is responsible for the initial digestion of food or the formation of a bolus, which occurs largely due to the presence of saliva and digestive enzymes secreted by the salivary glands [4]. The digestion of polysaccharides (starches) starts in the oral cavity due to the presence of enzyme a-amylase in salivary secretions. This amylase has also been known as ptyalin, but the starch digestive enzyme present in the oral cavity is now commonly known as salivary amylase [4]. The main carbohydrate-digesting enzyme present in the saliva is a-amylase which digests or breakdowns starch into maltose, maltotriose, and dextrine, and is inactivated by the acidic environment of the gastrointestinal tract [4]. Its biological function is to digest or break down starch into maltose, maltotriose, and dextrin. The majority of this enzyme is synthesized in the parotid glands and the remainder in the submandibular glands, contributing about $50 \%$ of the total salivary proteins produced by the glands [13]. Saliva in the oral cavity is typically the first point of contact with any ingested substance, and its composition may be influenced by medication, oral lesions, intracellular diffusion, proteolytic enzymes derived from the host, oral micro-organisms, exercise, and circadian patterns.

\section{Salivary diagnostics in clinical biochemistry}

As a result of the rapid progress in salivary studies, the concept of salivaomics, the analysis of proteins, RNA, DNA, lipids, carbohydrates, metabolites and microorganisms in saliva, is growing. It is now recognized that salivary biomolecules can assist in the early diagnosis of several oral and systemic diseases [18]. Developments in salivaomics have also led to recognition that saliva represents a pool of biomarkers. Whole saliva is a source of good diagnostic material and may serve as a substitute for blood in the monitoring, prognosis, and treatment of many diseases. Saliva contains a wide range of ingredients 
that reflect the level of biomarkers in real time as well as the composition of plasma. Saliva offers many advantages, including ease and noninvasive collection, with no risk of needle injuries apart from patient fear and cooperation. Moreover, saliva compounds are characterized by a relatively long shelf life compared with blood, and easier collection procedures may provide a cost-effective approach for screening large populations and eliminate the risk of contracting infectious diseases for medical staff and patients [19].

Saliva is a biofluid with a significant number of emerging applications in research and clinical settings. It can be used as an effective diagnostic tool for systemic conditions and several medications. Unlike other common biological fluids, such as blood and urine, saliva can be easily obtained, collected, and transported for analysis [20]. Studies investigating the use of saliva as a diagnostic fluid are not limited only to the diagnosis of oral diseases; many systemic diseases, such as different types of cancer, cardiovascular disease, immunological syndromes, and hereditary disorders can also be analyzed using salivary diagnostics [21]. Screening for viral infections based on the detection of specific antibodies against viruses (e.g., hepatitis, HIV) in mucosal transudate-enriched saliva is simple and accurate. Some tumor markers (e.g., c-erbB-e, p53 antigen, CA125) present in saliva may also be used for screening and early diagnosis of malignancies that appear in other parts of the body. Similarly, oral fluids are also a source of microbial or DNA data that may be useful for biomarker profiling of oral bacteria, systemic diseases, and forensic analysis [22]. A number of caries-risk assessment tests have been developed to measure salivary bacteria, such as Streptococci and Lactobacilli. Streptococci are associated with an increased risk of developing caries and Lactobacilli are found in individuals with higher carbohydrate consumption and are also associated with an increased risk of caries [23]. Salivary tests are useful indicators of caries susceptibility at the individual level where they can be used for caries prevention and to profile for disease susceptibility. The buffering capacity of saliva is a measure of the host's ability to neutralize the reduction of plaque $\mathrm{pH}$ produced by acidogenic bacteria and can be measured in saliva using a commercially available kit [23]. As biomarkers are generally present at very low concentrations in the saliva, the development of specific and sensitive analytical methods is needed. Despite these limitations, the interest in saliva as a diagnostic or screening medium has advanced in recent decades [24].

\section{Biochemistry of acute and chronic dental diseases}

The oral mucosa contains an elaborate immune system and preserves immunological homeostasis and a relative state of health despite the presence of microbes [25]. The etiological link between chronic inflammatory disease and systemic conditions suggests an association between periodontal health and cardiovascular disease, DM, pre-term and low birthweight neonates, and obesity [14]. The oral cavity is constantly exposed to unique tissue-specific signals, commensal microbes and their metabolites, tissue damage from mastication, and antigens from food and airborne particles [26]. Saliva also plays an important role in the regulation of oral mucosal immune reactions and the healing of mucosal lesions, wounds, and ulcers. There are numerous defense proteins present in the saliva, such as immunoglobulins, chaperokines, cationic peptides, lysozyme, bactericidal/permeability-increasing proteins, salivary amylase, cystatins, PRPs, mucins, peroxidases, statherin, and others involved in either innate or acquired immunity. The major protective functions of salivary secretions in the oral cavity are performed through immunological and non-immunological means in addition to direct antimicrobial activity [27-30].

The major changes to connective tissue in dental disease, especially pulpitis, gingivitis, and periodontitis, are the degradation of the extracellular matrix (ECM) due to the release of enzymes by host and bacterial cells, phagocytosis of matrix components, and release of cytokines, inflammatory mediators, and apoptotic proteins. The increased levels of biomolecules and enzymes cause dental inflammation, which signifies that host-derived biomarkers cause periodontal and inflammatory diseases [31]. Periodontitis is an inflammatory disease that causes tissue damage as a result of interaction between the host immune response and pathogenic bacteria. Development of dental plaque biofilm leads to the loss of periodontal attachment and can result in tooth loss. The etiopathogenesis involves various cellular pathways, including proinflammatory mediators such as growth factors, cytokines, and MMPs. MMPs are the most important components in tissue damage associated with periodontal disease due to their role in the breakdown of ECM. The pathogens in dental plaque stimulate host cells to release MMPs, leading to tissue damage [32]. Host cell-derived enzymes such as MMPs are an important group of proteinases associated with periodontal disease. Periodontal inflammation occurs in the gingival tissue in response to bacteria biofilm. Gingivitis is characterized by an initial increase in blood flow, enhanced vascular permeability, and the influx of cells (neutrophils and monocyte-macrophages) from the peripheral blood to the gingival crevice. These cells subsequently produce cytokines, such as interleukin (IL)-1 $\beta, \mathrm{IL}-6$, tumor necrosis factor alpha (TNF- $\alpha$ ), and immunoglobulins. The severity of periodontitis is associated with increases in IL-1 $\beta$, TNF- $\alpha$, prostaglandins (PE2) and MMPs, whereas their inhibition leads to a reduction in periodontal disease $[33,34]$. The oral mucosa protects the host against foreign antigens and pathogenic microorganisms through an elaborate immune system composed of numerous defense and protective proteins in the saliva that are involved in oral homeostasis, immunity, and tolerance.

\section{Laboratory diagnostics of disorders for dental treatment}

The use of laboratory diagnostics has expanded enormously in the past few decades due to the development of automat- 
ed analyzers and computerized systems. Treatment has improved via pathological investigations through routine tests ordered to monitor disease status, organ function, or the effect of medications. A typical laboratory report includes the patient's demographic data, date and time of specimen collection, the name of the consulting physician, and the results of pathological tests performed [35-37]. Many patients seek dental treatment when they are suffering or recovering from acute and chronic conditions or when experiencing the effects of medications. The laboratory report provides significant information regarding a patient's health that is beneficial in the diagnosis and management of oral diseases and dental treatment as well as the prognosis for a particular treatment. The information obtained from laboratory tests allows the clinician to make informed decisions regarding dental care and treatment modification to minimize complications. In addition to radiography, clinical laboratory investigations can help the dentist to diagnose oral disease, determine the severity of infection or modify medication use based on underlying diseases and proposed dental treatments [35-37].

Several diagnostic tools are available to physicians to assess patient blood glucose level. A fasting blood glucose analysis is typically used to measure the blood glucose level after an overnight fast and a postprandial sample is obtained after breakfast. The $\mathrm{HbA1c}$ measurement provides an estimation of the average blood glucose level over the past several months, which is used by physicians to evaluate and manage DM $[38,39]$. Several complications, such as periodontal disease, salivary gland dysfunction, halitosis, burning mouth sensation, and taste dysfunction have been associated with DM. Individuals with DM are more prone to fungal and bacterial infections, oral soft tissue lesions, compromised oral wound healing processes, dental caries, and tooth loss [40]. Therefore, it is important that dentists take hematological and biochemical parameters and their potential impact on oral health and hygiene into account. Before initiating treatment for a diabetic patient, dentists must consider the laboratory analysis to minimize the risk of a diabetic emergency and reduce the probability of oral complications. A dentist should also review the patient's current DM management plan, including the dose and time of medications, as well as any lifestyle modifications, such as exercise or nutritional changes. Dentists should be mindful of their patients' health record and medication use [41].

Laboratory investigations for bleeding disorders are advisable when an individual has a personal or family history of bleeding and laboratory findings suggest the possibility of an inherited or acquired bleeding disorder. It is often advantageous to simultaneously test for von Willebrand disease, platelet function disorders, and coagulation defects, including fibrinogen disorders. An investigation for rare bleeding disorders, such as those affecting factor XIII, a2 antiplasmin and plasminogen activator inhibitor-1, is appropriate when faced with a severe congenital or acquired bleeding disor- der that cannot be explained based on the results of initial diagnostic investigations. An organized strategy to investigate bleeding disorders considers the important issues, confirms abnormal findings, fosters proper interpretation of the results, and provides a helpful framework for assessing the cause of bleeding [42].

\section{Conclusion}

A thorough understanding of modern biochemistry and molecular biology will be useful to students of medicine, including but not limited to dentistry, and would allow greater applications of research and teaching. Hence, an important aspect in biochemistry instruction is an emphasis on the fact that the basic biochemical processes occurring in the oral cavity are the same as those taking place in other tissues and organs of the body. Saliva is secreted from the salivary glands and has multiple functions, including mouth cleansing and protection, antibacterial activity, and assisting digestion. With the rapid advancement in salivaomics, saliva is now well recognized as a source of biological markers, including DNA, RNA and proteins, and those in microbial biofilm. The major changes that occur in the connective tissue in cases of dental diseases, particularly pulpitis, gingivitis, and periodontitis, are the degradation of extracellular matrix due to the release of enzymes by host and bacterial cells, phagocytosis of matrix components and the release of cytokines, inflammatory mediators, and apoptotic proteins. The elevated levels of these molecules and enzymes reflect the degree of dental inflammation, indicating that host cell-derived molecules can lead to the development of periodontal and inflammatory diseases. Given the numerous possible oral manifestations of DM and the risk of a diabetic emergency, it is important for dentists to recognize and appreciate the impact of this disorder on dental care. A thorough understanding of DM and the implications for dental management are necessary for a dentist to provide optimal oral health care to diabetic patients. Testing for bleeding disorders is advisable when a patient presents with a personal or family history of bleeding and laboratory investigations suggest there may be an inherited or acquired bleeding disorder. An advanced laboratory investigation is helpful in the diagnosis of various blood disorders, including those associated with coagulation factors, clot stabilization, or fibrinolysis. Clinical and biomedical research has contributed greatly to advancements in dental and medical sciences. Medical and clinical biochemistry is becoming increasingly significant in dentistry, and should be recognized in both teaching and research.

Acknowledgement: This review article is dedicated in the memory of my late beloved father, one of the noblest and kindest human beings who lived and passed this side of the planet. The author wishes to acknowledge Prof. Fahim H. Khan and Prof. Waseem A. Siddiqui, leading researchers in biophysics and biochemistry at the Aligarh Muslim University, Aligarh for their 
encouragement, support and motivation. I also thank the editor and reviewer(s) for their critical acclaim and suggestions for the improvement of review article.

Conflict of Interest: None declared.

Financial Disclosure: None declared.

Peer-review: Externally peer-reviewed.

\section{References}

1. Guven Y. Scientific basis of dentistry. J Istanb Univ Fac Dent 2017;51(3):64-71. [CrossRef]

2. Dominiczak MH. Contribution of biochemistry to medicine: medical biochemistry and clinical biochemistry. Encyclopedia of life support systems (EOLSS). p. 1-9. Available at: https:// www.eolss.net/sample-chapters/C17/E6-58-10-12.pdf. Accessed Mar 16, 2021.

3. Loesche WJ. Microbiology of dental decay and periodontal disease. In: Baron S, editor. Medical microbiology. 4th ed. Galveston (TX): University of Texas Medical Branch at Galveston; 1996. Chapter 99.

4. Ahsan H. Biomolecules and biomarkers in oral cavity: bioassays and immunopathology. J Immunoassay Immunochem 2019;40(1):52-69. [CrossRef]

5. Kochhar A, Larian B, Azizzadeh B. Facial nerve and parotid gland anatomy. Otolaryngol Clin North Am 2016;49(2):27384. [CrossRef]

6. Ahsan $\mathrm{H}$. The role of biochemistry in dental sciences. J Med Erud 2014;2(2):43-9.

7. Gewargis J. The biochemical basis of dental caries in action. Acta Scientific Dental Sci 2019;3(3):32-6.

8. Beeley JA. The teaching of biochemistry to dental students. Biochem Edu 1974;2(1):9-11. [CrossRef]

9. Peng $Y$, Chen $X$, Sato T, Rankin SA, Tsuji RF, Ge Y. Purification and high-resolution top-down mass spectrometric characterization of human salivary a-amylase. Anal Chem 2012;84(7):3339-46. [CrossRef]

10. Spielmann N, Wong DT. Saliva: diagnostics and therapeutic perspectives. Oral Dis 2011;17(4):345-54. [CrossRef]

11. Pfaffe T, Cooper-White J, Beyerlein P, Kostner K, Punyadeera C. Diagnostic potential of saliva: current state and future applications. Clin Chem 2011;57(5):675-87. [CrossRef]

12. Chen YC, Li TY, Tsai MF. Analysis of the saliva from patients with oral cancer by matrix-assisted laser desorption/ionization time-of-flight mass spectrometry. Rapid Commun Mass Spectrom 2002;16(5):364-9. [CrossRef]

13. de Almeida Pdel V, Grégio AM, Machado MA, de Lima AA, Azevedo LR. Saliva composition and functions: a comprehensive review. J Contemp Dent Pract 2008;9(3):72-80. [CrossRef]

14. Acevedo AC. Saliva and oral health. Rev Assoc Med Bras 2010;56(1):2. [CrossRef]

15. Baron M, Norman DG, Campbell ID. Protein modules. Trends Biochem Sci 1991;16:13-7. [CrossRef]

16. Levine MJ. Salivary macromolecules. A structure/function synopsis. Ann N Y Acad Sci 1993;694:11-6. [CrossRef]
17. Schenkels LC, Veerman EC, Nieuw Amerongen AV. Biochemical composition of human saliva in relation to other mucosal fluids. Crit Rev Oral Biol Med 1995;6(2):161-75. [CrossRef]

18. Zhang CZ, Cheng XQ, Li JY, Zhang P, Yi P, Xu X, et al. Saliva in the diagnosis of diseases. Int J Oral Sci 2016;8(3):133-7. [CrossRef]

19. Zalewska A, Waszkiewicz N, López-Pintor RM. The use of saliva in the diagnosis of oral and systemic diseases. Dis Markers 2019;2019:9149503. [CrossRef]

20. Miočević O, Cole CR, Laughlin MJ, Buck RL, Slowey PD, Shirtcliff EA. Quantitative lateral flow assays for salivary biomarker assessment: a review. Front Public Health 2017;5:133. [CrossRef]

21. Podzimek S, Vondrackova L, Duskova J, Janatova T, Broukal Z. Salivary markers for periodontal and general diseases. Dis Markers 2016;2016:9179632. [CrossRef]

22. Fábián TK, Fejérdy P, Csermely P. Salivary genomics, transcriptomics and proteomics: the emerging concept of the oral ecosystem and their use in the early diagnosis of cancer and other diseases. Curr Genomics 2008;9(1):11-21. [CrossRef]

23. Whelton $\mathrm{H}$. Introduction: the anatomy and physiology of salivary glands. In: Edgar M, Dawes C, O'Mullane D, editors. Duns Tew, UK: Stephen Hancocks Limited; 2012.

24. Nunes LA, Mussavira S, Bindhu OS. Clinical and diagnostic utility of saliva as a non-invasive diagnostic fluid: a systematic review. Biochem Med (Zagreb) 2015;25(2):177-92. [CrossRef]

25. Hovav AH. Dendritic cells of the oral mucosa. Mucosal Immunol 2014;7(1):27-37. [CrossRef]

26. Moutsopoulos NM, Konkel JE. Tissue-Specific Immunity at the Oral Mucosal Barrier. Trends Immunol 2018;39(4):276-87.

27. Fábián TK, Fejérdy $P$, Nguyen MT, Soti $C$, Csermely P. Potential immunological functions of salivary $\mathrm{Hsp} 70$ in mucosal and periodontal defense mechanisms. Arch Immunol Ther Exp (Warsz) 2007;55(2):91-8. [CrossRef]

28. Fábián TK, Fejérdy $P, C$ sermely $P$. Saliva in health and disease. In: Begley TP, editor. Wiley encyclopedia of chemical biology. 1st ed. Hoboken, NJ: John Wiley \& Sons; 2008. vol. 4, p. 1-9.

29. Fábián TK, Gótai L, Beck A, Fábián TK, Fejérdy P. The role of molecular chaperones (Hspas/Hsp70s) in oral health and oral inflammatory diseases: a review. Eur J Inflamm 2009;7:53-61.

30. Fábián TK, Hermann P, Beck A, Fejérdy P, Fábián G. Salivary defense proteins: their network and role in innate and acquired oral immunity. Int J Mol Sci 2012;13(4):4295-320. [CrossRef]

31. Aksoy Y. Biochemical approach to dental diseases. Clin Dent Res 2011;35(1):57-64.

32. Checchi V, Maravic T, Bellini P, Generali L, Consolo U, Breschi $\mathrm{L}$, et al. The role of matrix metalloproteinases in periodontal disease. Int J Environ Res Public Health 2020;17(14):4923.

33. Taba M Jr, Kinney J, Kim AS, Giannobile WV. Diagnostic biomarkers for oral and periodontal diseases. Dent Clin North Am 2005;49(3):551-71, vi. [CrossRef]

34. Zia A, Khan S, Bey A, Gupta ND, Mukhtar-Un-Nisar S. Oral biomarkers in the diagnosis and progression of periodontal diseases. Biol Med 2011;3(2):45-52.

35. Hupp WS, Firriolo FJ, De Rossi SS. Laboratory evaluation of chronic medical conditions for dental treatment: part III. Hematology. Compend Contin Educ Dent 2011;32(7):10-2, 14-8; quiz 20, 32. 
36. Hupp WS, Firriolo FJ, DeRossi SS. Laboratory evaluation of chronic medical conditions for dental treatment, part I: HIV/AIDS, hemostasis, and liver disease. Inside Dentistry 2010;6(9):60-70.

37. Hupp WS, Firriolo FJ, DeRossi SS. Laboratory evaluation of chronic medical conditions for dental treatment, part II: diabetes and renal function. Inside Dentistry 2010:6(10);48-58.

38. Janghorbani M, Amini M. Normal fasting plasma glucose and risk of prediabetes and type 2 diabetes: the Isfahan diabetes prevention study. Rev Diabet Stud 2011;8(4):490-8. [CrossRef]
39. American Diabetes Association. Diagnosis and classification of diabetes mellitus. Diabetes Care 2011;34(Suppl 1):S62-9.

40. Al-Maskari AY, Al-Maskari MY, Al-Sudairy S. Oral manifestations and complications of diabetes mellitus: a review. Sultan Qaboos Univ Med J 2011;11(2):179-86. [CrossRef]

41. Miller A, Ouanounou A. Diagnosis, management, and dental considerations for the diabetic patient. J Can Dent Assoc 2020;86:k8.

42. Hayward CPM. How I investigate for bleeding disorders. Int J Lab Hematol 2018;40 Suppl 1:6-14. [CrossRef] 